\title{
Effect of selenite-ion on the integrity of winter rye seedlings (Secale cereale L.) membranes in drought conditions
}

\author{
Tamara Puzina*, Inna Makeeva, and Alina Nedbaylo \\ Orel State University named after I.S. Turgenev, 302026, city of Orel, Russia
}

\begin{abstract}
The effect of selenite-ion on the reactions of lipid peroxidation, the electrolyte release, the membrane damage coefficient and the growth parameters of winter rye seedlings of the Tatiana variety under optimal water supply conditions and during a seven-day drought was studied. Seeds treatment with $5.8 \cdot 10^{-3} \mathrm{M}$ sodium selenite solution reduced the accumulation of lipid peroxidation products in the leaves - lipid fatty acid hydroperoxides and malondialdehyde under optimal and stressful conditions. To a greater extent, selenite-ion reduced the content of malondialdehyde compared to hydroperoxides (by 25\%). The effect of selenium on lipid peroxidation reaction was more effective in drought conditions. The inhibition of electrolyte release from seedlings leaves enriched with selenium was revealed. The coefficient of membrane damage in drought conditions was lower in the variant with selenite-ion (by $37 \%$ ). The large mass of aboveground seedling organs, length and weight of the root system when treating seeds with sodium selenite were noted. The obtained data are discussed in connection with the effect of selenium on the content of auxins, expressing genes responsible for the antioxidant enzymes synthesis.
\end{abstract}

\section{Introduction}

Stressful environmental conditions cause overproduction of reactive oxygen species, which leads to modification of the structure of not only proteins and DNA, but also membranes. Under these conditions, reactive oxygen species provoke a chain process of membrane destruction caused by lipid peroxidation [1]. As a result, their properties and functions are violated. The plant antioxidant system does not always cope with the elimination of reactive oxygen species. Therefore, in the practice of crop production, exogenous antioxidants are used. Among their diversity, a special role is given to the trace element - selenium [2]. The data available in the literature mainly concern the effect of selenium on the activity of antioxidant enzymes and plant productivity in stressful conditions $[3 ; 4]$. Only in some studies, its effect on the accumulation of lipid peroxidation products is noted, the content of which can be used to evaluate the integrity of membranes under stress [5]. No information was found on the participation of selenium in the regulation of LPO reactions and the preservation of membrane integrity in rye plants under conditions of water

*Corresponding author: tipuzina@gmail.com 
scarcity. It is known that at the first stages of development, rye seedlings, especially winter varieties, are very demanding to moisture [6].

The aim of this work was to study the effect of selenite-ion on the electrolyte release and the lipid peroxidation reaction in winter rye seedlings under drought conditions.

\section{Materials and methods}

The object of the study was of winter rye (Secale cereale L.) seedlings of the Tatiana variety selected by the FSBRI Federal Research Center Nemchinovka.

The plants were grown in laboratory conditions in containers with soil "Universal for seedlings" at a temperature of $18 \pm 2^{\circ} \mathrm{C}$.

Variants of the experiment included rye seeds soaking in a $5.8 \cdot 10^{-3} \mathrm{M}$ solution of sodium selenite containing $0.0026 \mathrm{mM}$ of selenium. The control seeds were soaked in water. Drought in 10-day-old seedlings was created by stopping watering for seven days (soil moisture content is $40 \%$ of the total moisture capacity).

For analysis, average samples of leaves and roots were taken in 17-day-old seedlings.

The electrolyte release was determined by the electrical conductivity of the solution with a leaf sample after a 4-hour exposure and after a 20-minute boiling (full electrolyte release) using the Expert 003 conductometer (Russia) according to the method [7]. The membrane damage coefficient was calculated by the formula:

$$
\mathrm{DC}=\frac{L_{D}-L_{0}}{100-L_{0}} \cdot 100 \%
$$

where $L_{0}$ is the electrolyte release under optimal water supply conditions ( $\%$ of the total release during boiling), $L_{D}$ is the electrolyte release during drought.

The content of lipid fatty acids hydroperoxides was estimated by the reaction of their interaction with ammonium rhodanide [8]. The content of malonic dialdehyde was determined by the color reaction with thiobarbituric acid under heating [9].

The mass of leaves and roots was determined by weighing on an electronic scale VST ("Ves-Service", city of St. Petersburg).

The figures show the arithmetic averages of the five biological and five analytical repetitions and their standard errors. The reliability of the results was evaluated using the Student's test, considering the differences to be significant when the confidence level is higher than 0.95 .

\section{Results and discussion}

The effect of drought on rye plants in comparison with other grain crops and, above all, wheat, is almost not studied since many of its varieties are drought-resistant with the exception of winter crops.

One of the indicators of membrane integrity in stressful conditions is the electrolyte release [10]. In optimal water supply conditions, selenite-ion significantly reduced the electrolyte release at 4-hour exposure of the leaves in distilled water by $14 \%$ against the control, and at 20-minute boiling, when complete destruction of the membranes occurred, by $19 \%$ (Fig. 1 a, b). Under the conditions of a 7-day drought, the regularity in the sodium selenite action on the electrolyte release from the leaves remained. At the same time, it is important to note that the effect of selenite-ion on the inhibition of this process was $26 \%$ higher under the conditions of the stressor action against the optimal water supply conditions both before and after boiling. 
(a)

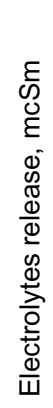

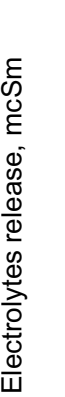

0

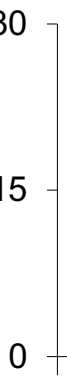

\section{(a)}

政

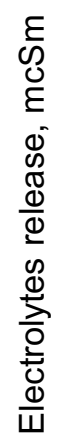

(a)

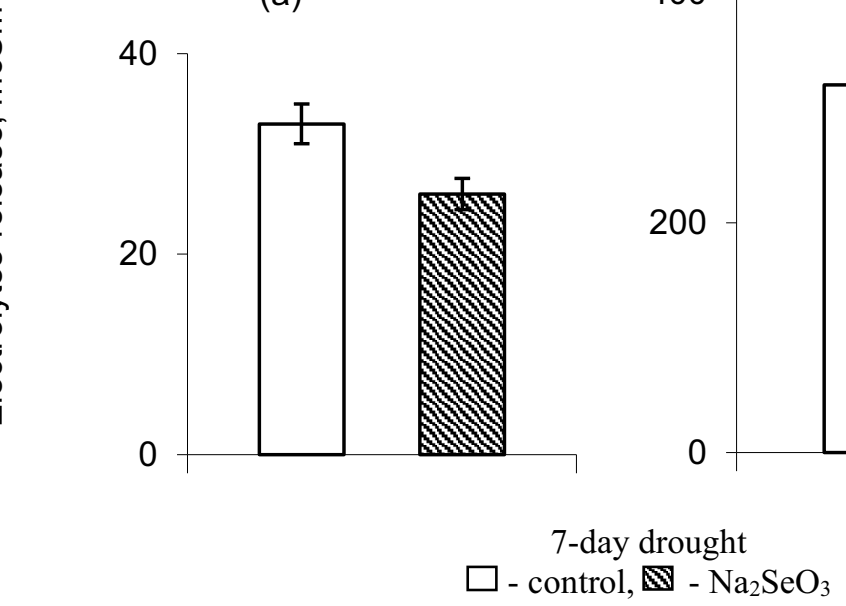

300

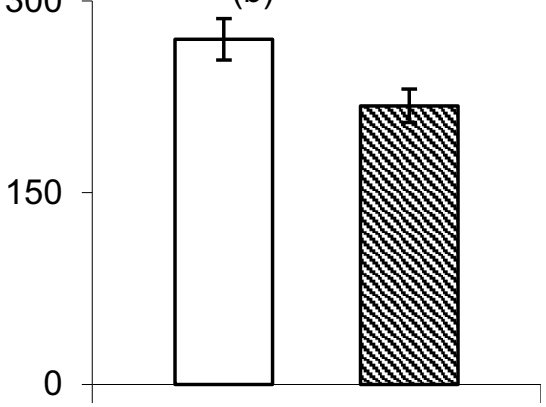

Optimal water supply conditions

Fig. 1. The effect of selenite-ion on the electrolyte release from leaves under optimal water supply conditions and during drought. ( $\mathrm{a}$ - before boiling; $\mathrm{b}$ - after boiling.)

The calculation of the membrane damage coefficient during drought in rye seedlings most clearly showed the protective role of selenium (Fig. 2). Specifically, the enrichment of seedlings with selenium reduced membrane damage by $37 \%$ in these stressful conditions.

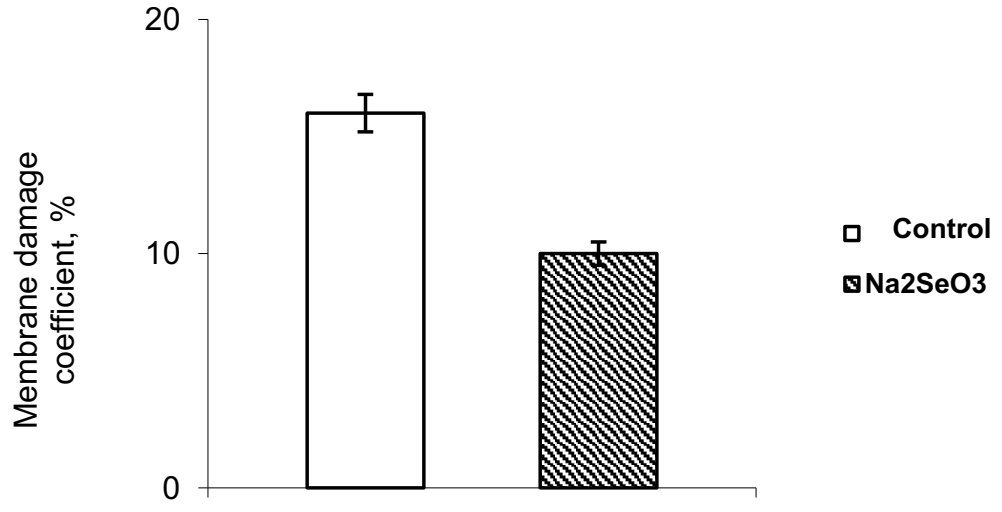

Fig. 2. The effect of selenite-ion on the membrane integrity in drought conditions. 
One of the reasons for the increase in the permeability of membranes for electrolytes is the change in the phase state of the membrane bilayer under the influence of lipid peroxidation [11]. In this regard, the positive effect of selenium on the preservation of the integrity of rye seedling membranes can be associated with the inhibition of lipid peroxidation reactions. As a test for the intensity of lipid peroxidation, the content of the final product of this process - malondialdehyde - is most often used. Our monitoring of lipid peroxidation reactions not only for the final product - malonic dialdehyde, but also for hydroperoxides of lipid fatty acids - primary products, showed that the content of hydroperoxides under the influence of selenite-ion decreased by $16 \%$ under optimal water supply conditions (Fig.3). The drought activated the lipid peroxidation reactions. In this way, in the control - by $21 \%$, while in the variant with selenium - by $14 \%$. As a result, under stress conditions, the selenium effect was higher compared to stress-free conditions. The decrease in the accumulation of hydroperoxides was $21 \%$. The drought caused the accumulation of malondialdehyde in the seedlings in the control variant. The increase was $33 \%$.

The effect of selenium enrichment on the content of malondialdehyde was similar to the change in the content of hydroperoxides (Fig. 3). It can only be noted that selenium reduced the accumulation of malondialdehyde to a greater extent both under optimal water supply conditions and during drought compared to hydroperoxides (by 25\%). According to the literature [12], other stressors, such as the xenobiotic paraquat, hypo- and hyperthermia, also cause an increase in the formation of malondialdehyde in young rye plants.
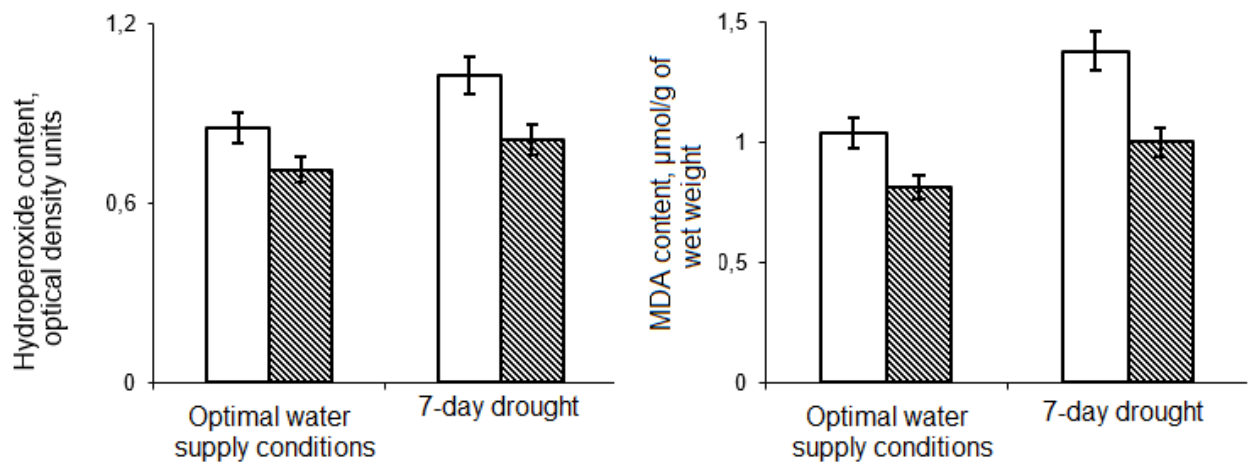

control, $-\mathrm{Na}_{2} \mathrm{SeO}_{3}$

Fig. 3. The effect of selenite-ion on the lipid peroxidation reactions under optimal water supply conditions and in drought.

A decrease in the accumulation of lipid peroxidation products under the influence of selenite-ion may be associated with the activation of antioxidant enzymes against the background of an increase in the content of auxins phytohormones. Such information was obtained earlier on the example of potato plants [13]. Auxins are known to cause the expression of superoxide dismutase and catalase genes $[14 ; 15]$.

Against the background of a decrease in the activity of lipid peroxidation reactions and the coefficient of membrane damage under the influence of selenite-ion in drought conditions, an increase in the growth parameters of rye seedlings was revealed in comparison with the control variant (Fig. 4). Thus, the mass of aboveground organs was higher by $23 \%$, the mass of roots - by $27 \%$, the length of roots - by $29 \%$. 

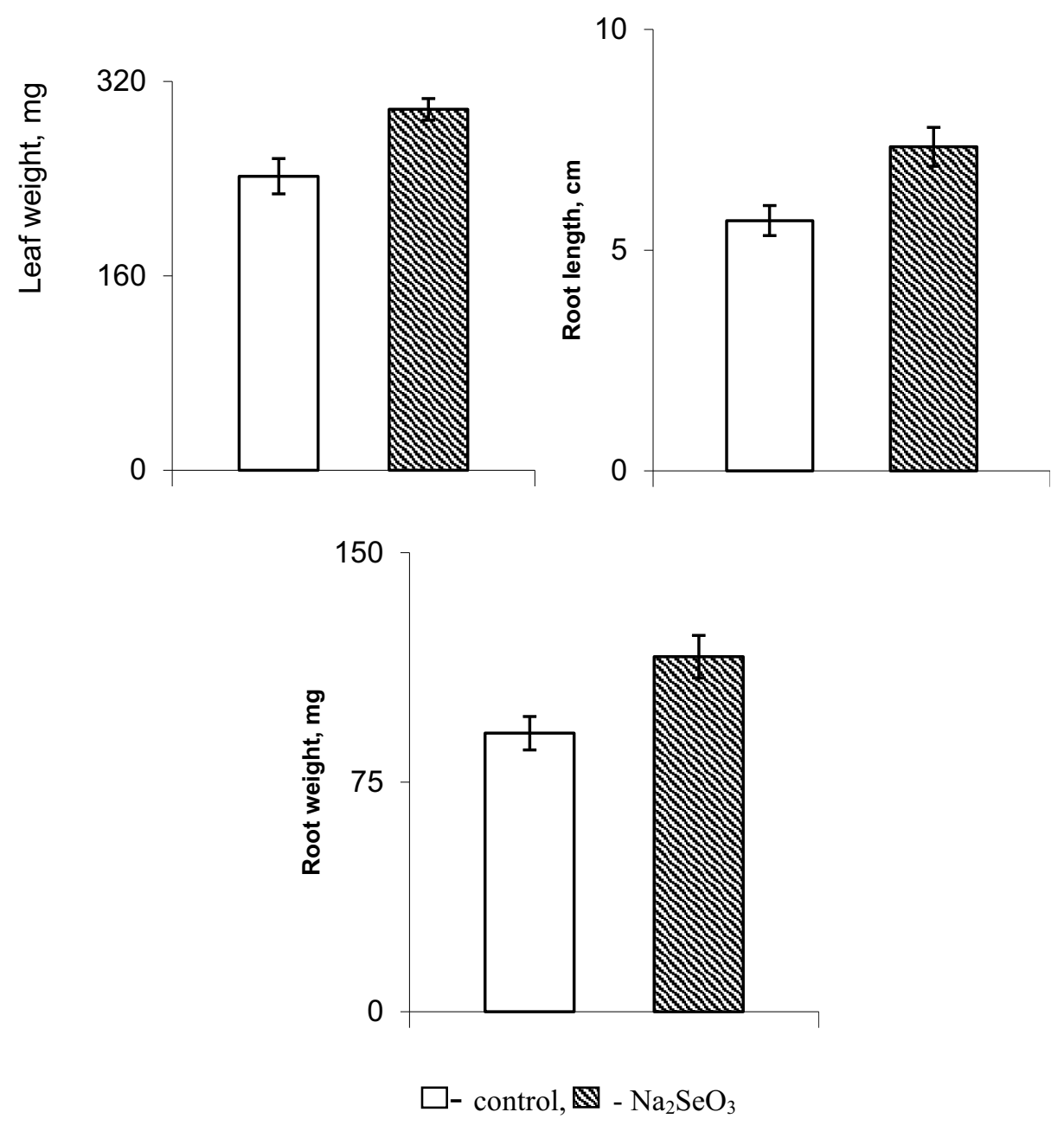

Fig. 4. The effect of selenite-ion on the growth parameters of rye seedlings in drought conditions.

\section{Conclusion}

Monitoring of the formation of the primary and final product of lipid peroxidation revealed their accumulation in the leaves of winter rye seedlings in the 7-day drought. Pretreatment of seeds with sodium selenite inhibited the formation of both fatty acid hydroperoxides and malondialdehyde, and the latter to a greater extent. The protective effect of selenite-ion on the state of the lipid bilayer of the membranes affected the preservation of their integrity, as indicated by a decrease in the electrolyte release and the calculated coefficient of damage to the membranes during drought. In these conditions, a slightly greater growth activity of the rye seedlings organs was noted in comparison with the control.

\section{References}

1. N.M. Hassan, M.M. Nemat Alla, Acta Physiol Plant, 27, 429 (2005)

2. P.A. Poluboyarinov, N.A. Golubkina, Russian Journal of Plant Physiology, 62(3), 367 (2015) 
3. I.I. Seregina, N.T. Nilovskaya, N.V. Ostapenko, Agrochemistry, 1, 44 (2001)

4. Vas.V. Kuznetsov, V.P. Kholodova, Vl.V. Kuznetsov, B.A. Yagodin, Report of AS, 390, 713 (2003)

5. T.I. Puzina, M.A. Tsukanova, Scientific Notes of the Orel State University. Natural, Technical and Medical Sciences Series, 2, 51 (2008)

6. A.A. Zhuchenko, Agri-food Policy, 3, 14 (2012)

7. Henry A. Hepburn, Bernard A. Goodman, Donald B. McPhail, Stan Matthews, Alison A. Powell, Journal of Experimental Botany, 37(11), 1675(1986)

8. L.A. Romanova, I.D. Stalnaya, Modern methods in biochemistry, 65 (1977)

9. A.S. Lukatkin, Journal of Plant Physiology, 49(6), 878 (2002)

10. A.S. Lukatkin, N.N. Kashtanova, P. Dukhovskis, Reports of the Russian Academy of Agricultural Sciences, 3, 16 (2013)

11. T. Senaratna, J.F. Gusse and B.D. McKersie, Physiologia Plantarum, 73, 85 (1988)

12. A.S. Semenova, A.S. Lukatkin, Russ. J. Plant Physiol., 62, 797 (2015)

13. T.I. Puzina, N.S. Vlasova, I.Yu. Makeeva, V.L. Lantsev, Cell and Tissue Biology, 58 (7), 555 (2016)

14. A.P. Veselov, L.N. Kurganova, A.A. Brilkina, Herald of UNN. Ser. Biol., 1, 159 (2001)

15. L.M. Guan, J.G. Scandalios, Physiologia Plantarum, 114, 288 (2002) 